\title{
Quadratic Stochastic Euclidean Bipartite Matching Problem
}

\author{
Sergio Caracciolo* \\ Dipartimento di Fisica, University of Milan and INFN, via Celoria 16, I-20133 Milan, Italy \\ Gabriele Sicuro ${ }^{\dagger}$ \\ Centro Brasileiro de Pesquisas Físicas, Rua Xavier Sigaud 150, 22290-180 Rio de Janeiro, Rio de Janeiro, Brazil
}

(Received 9 October 2015; published 1 December 2015)

\begin{abstract}
We propose a new approach for the study of the quadratic stochastic Euclidean bipartite matching problem between two sets of $N$ points each, $N \gg 1$. The points are supposed independently randomly generated on a domain $\Omega \subset \mathbb{R}^{d}$ with a given distribution $\rho(\mathbf{x})$ on $\Omega$. In particular, we derive a general expression for the correlation function and for the average optimal cost of the optimal matching. A previous ansatz for the matching problem on the flat hypertorus is obtained as a particular case.
\end{abstract}

The Euclidean bipartite matching problem (EBMP) was firstly introduced and studied by Monge [1] in 1781. It is an assignment problem in which an underlying geometric structure is present. Assignment problems are of paramount importance in theoretical computer science [2,3] and a polynomial-time algorithm, the celebrated Hungarian algorithm [4-6], is available for their solution. In the EBMP two sets of $N$ points, let us call them $\mathcal{R}:=\left\{\mathbf{r}_{i}\right\}_{i=1, \ldots, N}$ and $\mathcal{B}:=\left\{\mathbf{b}_{i}\right\}_{i=1 \ldots \ldots N}$, are considered on a domain $\Omega \subseteq \mathbb{R}^{d}$ in $d$ dimensions. The problem, in its quadratic version, asks for the permutation $\pi \in \mathcal{S}_{N}, \mathcal{S}_{N}$ symmetric group of $N$ elements, such that the cost functional

$$
E_{N}[\pi]:=\frac{1}{N} \sum_{i=1}^{N}\left\|\boldsymbol{\mu}_{\pi(i)}(i)\right\|^{2}
$$

is minimized. In the previous formula we have introduced

$$
\boldsymbol{\mu}_{j}(i):=\mathbf{b}_{j}-\mathbf{r}_{i}
$$

and we have denoted by $\|\bullet\|$ the Euclidean norm in $\mathbb{R}^{d}$.

Matching problems appear in many different physical, biological, and computational applications. The (linear) EBMP, for example, was introduced by Monge to optimize the transport cost of soil from $N$ mining sites to $N$ construction sites. The problem of covering a given lattice with dimers can also be reformulated as a matching problem [7], whereas, in computational biology, matching techniques are applied to pattern recognition problems [8]. In the computer vision, the quadratic EBMP is at the basis of many image stitching and stereographic reconstruction algorithms [9]. Finally, the quadratic cost functional in Eq. (1) plays a special role in physical applications. Indeed, it was used by Tanaka [10] in the study of the Boltzmann equation, and by Brenier [11] in his variational formulation of Euler incompressible fluids.
In many applications, however, the parameters (for example, the positions of the points) are affected by uncertainty, and the matching problem is a stochastic (or random) optimization problem. In this case, the average properties of the solution are of some interest.

Many analytical and numerical techniques, derived from statistical physics $[12,13]$, were successfully applied to the study of stochastic optimization problems. In particular, in the random assignment problem (RAP), the quantities $\left\|\boldsymbol{\mu}_{j}(i)\right\|$ are considered independent and identically distributed random variables and the Euclidean structure is completely neglected. The RAP was one of the first stochastic optimization problems to be solved using the theory of disordered systems by Mézard and Parisi [14]. Their results, obtained for $N \rightarrow \infty$, were rigorously derived years later by Aldous [15]. Subsequently, Linusson and Wästlund [16] and Nair et al. [17], in two remarkable papers, proved independently Parisi's conjecture [18] about the average optimal cost at finite $N$. They were able to prove also the more general Coppersmith-Sorkin conjecture [19] regarding the average optimal cost in the so-called $k$-assignment problem.

In the present Letter we deal with the stochastic EBMP (sEBMP). In the sEBMP the two sets of $N$ elements, $\mathcal{R}$ and $\mathcal{B}$, respectively (the instance of the problem), are obtained extracting $2 N$ points independently with a given probability distribution density $\rho(\mathbf{x})$ on the domain $\Omega$. We are interested in the average properties of the optimal matching, and, in particular, in the optimal matching cost and in correlation functions. In contrast with the RAP, in our case a Euclidean correlation appears among different values $\left\|\boldsymbol{\mu}_{j}(i)\right\|$. This correlation is due to the underlying geometric structure. Denoting by $\overline{\boldsymbol{}}$ the average over all instances, the average optimal cost (AOC) is

$$
E_{N}:=\overline{\min _{\pi} E_{N}[\pi]} .
$$

This problem was studied perturbatively, under the assumptions $\rho(\mathbf{x})=1$ and $\Omega \equiv[0,1]^{d}$, by Mézard and Parisi [20], 
using the RAP as a mean field approximation. Their predictions were later confirmed numerically [21,22]. In Ref. [23] a proper scaling ansatz was adopted to evaluate directly the AOC and its finite size correction in any dimension, assuming a uniform distribution on the hypertorus. The one dimensional problem, again under the hypothesis of uniform distribution, was exactly solved in Refs. [24,25].

Inspired by the celebrated Monge-Kantorovič theory of optimal transportation [26,27], we propose here a very general framework for the solution of the problem. Under the hypothesis that the points are generated using the same probability distribution density, we can indeed write down a quadratic functional in the large $N$ limit. This functional can be used to compute every correlation function of the optimal solution of the quadratic sEBMP and to evaluate the scaling of the AOC.

Let us first consider a bounded $d$-dimensional domain $\Omega \subset \mathbb{R}^{d}$. Let $\mathcal{R}:=\left\{\mathbf{r}_{i}\right\}_{i=1, \ldots, N}$ and $\mathcal{B}:=\left\{\mathbf{b}_{i}\right\}_{i=1, \ldots, N}$, be two sets, each one consisting of $N$ points independently generated with the same probability distribution density $\rho(\mathbf{x})>0$ on $\Omega \backslash \partial \Omega, \partial \Omega$ boundary of $\Omega$. We introduce the following empirical measures:

$$
\begin{gathered}
\rho_{\mathcal{R}}(\mathbf{x}):=\frac{1}{N} \sum_{i=1}^{N} \delta^{(d)}\left(\mathbf{x}-\mathbf{r}_{i}\right), \\
\rho_{\mathcal{B}}(\mathbf{x}):=\frac{1}{N} \sum_{i=1}^{N} \delta^{(d)}\left(\mathbf{x}-\mathbf{b}_{i}\right) .
\end{gathered}
$$

We define also the functional

$$
E[\boldsymbol{\mu}]:=\int_{\Omega}\|\boldsymbol{\mu}(\mathbf{x})\|^{2} \rho_{\mathcal{R}}(\mathbf{x}) d \mathbf{x}
$$

for a map $\mu: \Omega \rightarrow \mathbb{R}^{d}$. The previous functional provides a correct matching cost, Eq. (1), if, and only if, $\boldsymbol{\mu}\left(\mathbf{r}_{i}\right)=$ $\mathbf{b}_{\pi(i)}-\mathbf{r}_{i}$ for a certain permutation $\pi \in \mathcal{S}_{N}$. This additional constraint implies

$$
\int_{\Omega} \delta^{(d)}[\mathbf{x}-\mathbf{y}-\boldsymbol{\mu}(\mathbf{y})] \rho_{\mathcal{R}}(\mathbf{y}) d \mathbf{y}=\rho_{\mathcal{B}}(\mathbf{x})
$$

We can write down a "partition function" for our problem introducing a proper Lagrange multiplier $\varphi(\mathbf{x})$ to impose the constraint in Eq. (6),

$$
Z(\beta) \propto \int[\mathcal{D} \boldsymbol{\mu}] \int_{-i \infty}^{+i \infty}[\mathcal{D} \varphi] e^{-\beta S[\boldsymbol{\mu}, \varphi]},
$$

the optimal solution being recovered for $\beta \rightarrow+\infty$. The exponent in the functional integral is

$$
\begin{aligned}
S[\boldsymbol{\mu}, \varphi]: & =\frac{1}{2} E[\boldsymbol{\mu}]+\int_{\Omega}\left\{\varphi(\mathbf{x}) \rho_{\mathcal{B}}(\mathbf{x})-\varphi[\mathbf{x}+\boldsymbol{\mu}(\mathbf{x})] \rho_{\mathcal{R}}(\mathbf{x})\right\} d \mathbf{x} \\
= & -\int_{\Omega}\left[\varphi(\mathbf{x}) \varrho(\mathbf{x})+\rho_{\mathcal{R}}(\mathbf{x}) \boldsymbol{\mu}(\mathbf{x}) \cdot \nabla \varphi(\mathbf{x})\right] d \mathbf{x} \\
& +\frac{1}{2} E[\boldsymbol{\mu}]+s[\boldsymbol{\mu}, \varphi],
\end{aligned}
$$

where $s[\boldsymbol{\mu}, \varphi]=O\left(\|\boldsymbol{\mu}\|^{2} \varphi\right)$ are higher order nonlinear terms in the fields obtained from the Taylor series expansion of $\varphi(\mathbf{x}+\boldsymbol{\mu})$ around $\boldsymbol{\mu}=\mathbf{0}$. We introduced also

$$
\varrho(\mathbf{x}):=\rho_{\mathcal{R}}(\mathbf{x})-\rho_{\mathcal{B}}(\mathbf{x}) .
$$

Observing that $\rho_{\mathcal{R}}(\mathbf{x})$ is almost surely zero everywhere on the boundary, the Euler-Lagrange equations are

$$
\begin{aligned}
\varrho(\mathbf{x}) & =\nabla \cdot\left[\rho_{\mathcal{R}}(\mathbf{x}) \boldsymbol{\mu}(\mathbf{x})\right]-\frac{\delta s[\boldsymbol{\mu}, \varphi]}{\delta \varphi(\mathbf{x})}, \\
\rho_{\mathcal{R}}(\mathbf{x}) \boldsymbol{\mu}(\mathbf{x}) & =\rho_{\mathcal{R}}(\mathbf{x}) \nabla \varphi(\mathbf{x})-\frac{\delta s[\boldsymbol{\mu}, \varphi]}{\delta \boldsymbol{\mu}(\mathbf{x})}
\end{aligned}
$$

It is well known that in the $N \rightarrow \infty$ limit, the empirical measures $\rho_{\mathcal{R}}(\mathbf{x})$ and $\rho_{\mathcal{B}}(\mathbf{x})$ both converge (in a weak sense) to $\rho(\mathbf{x})$. In this limit the optimal field $\boldsymbol{\mu}^{*}$ is trivially $\boldsymbol{\mu}^{*}(\mathbf{x}) \equiv \mathbf{0} \forall \mathbf{x} \in \Omega$. For $N \gg 1$ we expect that the relevant contribution is given by small values of $\|\boldsymbol{\mu}\|$ and the nonlinear terms in $s$ are higher order corrections to the leading quadratic terms. The saddle point equations simplify as

$$
\begin{aligned}
& \varrho(\mathbf{x})=\nabla \cdot[\rho(\mathbf{x}) \boldsymbol{\mu}(\mathbf{x})], \\
& \boldsymbol{\mu}(\mathbf{x})=\nabla \varphi(\mathbf{x}) .
\end{aligned}
$$

The strict analogy between our problem and an electrostatic problem is evident. The field $\boldsymbol{\mu}$ plays the role of an electric field $\mathbf{E},-\varphi$ is the scalar potential, and, indeed, it acts as a Lagrange multiplier which implements the Gauss law, whereas $\rho$ corresponds to a dielectric function $\epsilon$ in a linear dielectric medium, in such a way that the equivalent of the displacement field $\mathbf{D}=\epsilon \mathbf{E}$ is $\rho \nabla \varphi$. The $\mathcal{B}$ points and the $\mathcal{R}$ points play the role of pointlike charges of opposite sign, being the overall charge $\int_{\Omega} \varrho(\mathbf{x}) d \mathbf{x}=0$. It is remarkable that Eq. (11b) reproduces the known result in measure theory that the transport field is a gradient [26] but, in our approach, this is specified as the gradient of the introduced Lagrange multiplier. We impose Neumann boundary conditions

$$
\left.\left.\nabla_{\mathbf{n}(\mathbf{x})} \varphi(\mathbf{x})\right|_{\mathbf{x} \in \partial \Omega} \equiv \nabla \varphi(\mathbf{x}) \cdot \mathbf{n}(\mathbf{x})\right|_{\mathbf{x} \in \partial \Omega}=0
$$

where $\mathbf{n}(\mathbf{x})$ is the normal unit vector to the boundary in $\mathbf{x} \in \partial \Omega$. This condition guarantees that the shape of the boundary is not modified in the $N \rightarrow \infty$ limit. We can therefore compute $\varphi$ as the solution of the following equation on $\Omega$ with the given boundary conditions 


$$
\nabla \cdot[\rho(\mathbf{x}) \nabla \varphi(\mathbf{x})]=\varrho(\mathbf{x}) .
$$

To solve Eq. (13), we use the modified Green's function $G_{\rho}(\mathbf{x}, \mathbf{y})$ of the operator $\nabla \cdot[\rho(\mathbf{x}) \nabla \bullet]$ on $\Omega$, defined by

$$
\begin{aligned}
& \nabla_{\mathbf{x}} \cdot\left[\rho(\mathbf{x}) \nabla_{\mathbf{x}} G_{\rho}(\mathbf{x}, \mathbf{y})\right]=\delta^{(d)}(\mathbf{x}-\mathbf{y})-\frac{1}{|\Omega|}, \\
& \text { with }\left.\frac{\partial G_{\rho}(\mathbf{x}, \mathbf{y})}{\partial \mathbf{n}(\mathbf{x})}\right|_{\mathbf{x} \in \partial \Omega}=0 .
\end{aligned}
$$

In Eq. (14), $|\Omega|$ is the Lebesgue measure of $\Omega$. We can write an explicit expression for $\boldsymbol{\mu}(\mathbf{x})$ as

$$
\boldsymbol{\mu}(\mathbf{x})=\int_{\Omega} \nabla_{\mathbf{x}} G_{\rho}(\mathbf{x}, \mathbf{y}) \varrho(\mathbf{y}) d \mathbf{y} .
$$

Averaging over the disorder, we easily obtain the following two-point correlation function

$$
\begin{aligned}
& C(\mathbf{x}, \mathbf{y}) \\
& :=\overline{\boldsymbol{\mu}(\mathbf{x}) \cdot \boldsymbol{\mu}(\mathbf{y})} \\
& =\iint_{\Omega_{N}(\mathbf{x}) \times \Omega_{N}(\mathbf{y})}\left[\overline{\varrho(\mathbf{z}) \varrho(\mathbf{w})} \nabla_{\mathbf{x}} G_{\rho}(\mathbf{x}, \mathbf{z}) \cdot \nabla_{\mathbf{y}} G_{\rho}(\mathbf{y}, \mathbf{w})\right] d \mathbf{z} d \mathbf{w} \\
& =\frac{2}{N} \int_{\Omega_{N}(\mathbf{x}, \mathbf{y})}\left[\rho(\mathbf{z}) \nabla_{\mathbf{x}} G_{\rho}(\mathbf{x}, \mathbf{z}) \cdot \nabla_{\mathbf{y}} G_{\rho}(\mathbf{y}, \mathbf{z})\right] d \mathbf{z} \\
& -\frac{2}{N} \iint_{\Omega_{N}(\mathbf{x}) \times \Omega_{N}(\mathbf{y})}\left[\rho(\mathbf{z}) \rho(\mathbf{w}) \nabla_{\mathbf{x}} G_{\rho}(\mathbf{x}, \mathbf{z}) \cdot \nabla_{\mathbf{y}} G_{\rho}(\mathbf{y}, \mathbf{w})\right] d \mathbf{z} d \mathbf{w},
\end{aligned}
$$

where we denoted by $\overline{\boldsymbol{}}$ the average over all instances. In the previous equation we used the following result

$$
\overline{\varrho(\mathbf{z}) \varrho(\mathbf{w})}=2 \frac{\rho(\mathbf{z})}{N}\left[\delta^{(d)}(\mathbf{z}-\mathbf{w})-\rho(\mathbf{w})\right] .
$$

Moreover, we introduced a proper cutoff to avoid divergences in the expression $N C(\mathbf{x}, \mathbf{y})$ and take into account finite size effects. This cutoff has indeed an intuitive explanation. Let $\delta_{N}$ be the scaling law in $N$ of the average distance between two nearest neighbor points randomly generated on $\Omega$ accordingly to $\rho(\mathbf{x})$. We introduced

$$
\begin{aligned}
\Omega_{N}(\mathbf{x}): & =\left\{\mathbf{y} \in \Omega:\|\mathbf{x}-\mathbf{y}\|>\alpha \delta_{N}\right\}, \\
\Omega_{N}(\mathbf{x}, \mathbf{y}):= & \left\{\mathbf{z} \in \Omega:\|\mathbf{x}-\mathbf{z}\|>\alpha \delta_{N}\right. \\
& \text { and } \left.\|\mathbf{y}-\mathbf{z}\|>\alpha \delta_{N}\right\}, \quad \alpha \in \mathbb{R}^{+} .
\end{aligned}
$$

Observe that $\delta_{N} \stackrel{N \rightarrow \infty}{\longrightarrow} 0$. The scaling quantity $\delta_{N}$ takes into account the nonzero characteristic length for finite $N$. The results of the computation may depend upon the regularizing parameter $\alpha$.
Equation (16) provides a recipe for the calculation of the AOC and for the correlation function in the sEBMP. In particular, in our approximation we have that

$$
E_{N} \simeq \int_{\Omega} C(\mathbf{x}, \mathbf{x}) \rho(\mathbf{x}) d \mathbf{x}
$$

If no regularization is required $(\alpha=0)$ we can write

$$
E_{N} \simeq \frac{2}{N} \iint_{\Omega \times \Omega} \rho(\mathbf{x})\left[\rho(\mathbf{y}) G_{\rho}(\mathbf{x}, \mathbf{y})-\frac{G_{\rho}(\mathbf{x}, \mathbf{x})}{|\Omega|}\right] d \mathbf{x} d \mathbf{y} .
$$

Let us now consider the one dimensional problem, $\Omega=[a, b] \subset \mathbb{R}$, and a certain probability density distribution $\rho(x)$ on $\Omega$. In this case we can explicitly write $(\alpha=0)$ the correlation function and the AOC, from Eq. (16) and Eq. (19) respectively. Imposing Neumann boundary conditions $\left.\partial_{x} \varphi(x)\right|_{x=a}=\left.\partial_{x} \varphi(x)\right|_{x=b}=0$,

$$
\begin{aligned}
C(x, y) & =\frac{2}{N} \frac{\Phi_{\rho}(\min \{x, y\})-\Phi_{\rho}(x) \Phi_{\rho}(y)}{\rho(x) \rho(y)}, \\
E_{N} & =\frac{2}{N} \int_{a}^{b} \frac{\Phi_{\rho}(x)\left[1-\Phi_{\rho}(x)\right]}{\rho(x)} d x,
\end{aligned}
$$

where we introduced the cumulative function

$$
\Phi_{\rho}(x):=\int_{a}^{x} \rho(\xi) d \xi .
$$

Our approach is suitable for many applications. In the following, we shall provide some examples and numerical verifications.

Matching problem on the interval.-As an application of Eqs. (21), let us assume, for example, a semicircle distribution on $\Omega \equiv[-1,1]$,

$$
\begin{gathered}
\rho(x)=\frac{2 \sqrt{1-x^{2}}}{\pi} \quad x \in[-1,1], \\
\Phi_{\rho}(x)=1+\frac{x \sqrt{1-x^{2}}-\arccos x}{\pi} .
\end{gathered}
$$

We can compute straightforwardly the correlation function and the AOC using Eqs. (21). In particular, we obtain the nontrivial result

$$
E_{N}=\frac{1}{N}\left(\frac{\pi^{2}}{6}-\frac{5}{8}\right)+o\left(\frac{1}{N}\right) .
$$

In Fig. 1 we compare the numerical results with the analytical predictions, showing the excellent agreement both for the correlation function and for the AOC.

Observe also that Eq. (21a) provides the correct correlation function for the sEBMP on $\Omega \equiv[0,1]$ with uniform distribution. Assuming indeed $\rho(x)=\theta(x) \theta(1-x)$, being $\theta(x)$ the Heaviside function, we have 


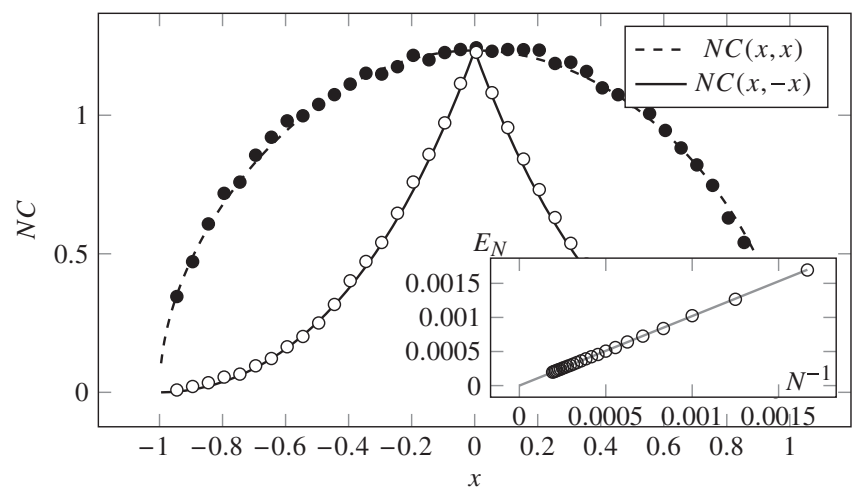

FIG. 1. sEBMP on the real line with points generated using a semicircle distribution, Eqs. (23). We plot the correlation functions $C(x, x)$ and $C(x,-x)$ for $N=3000$, obtained averaging over 5000 instances of the problem. We plot also the AOC $E_{N}$ obtained averaging over 5000 instances. We compare with the theoretical predictions obtained from Eqs. (21).

$$
\begin{aligned}
C(x, y) & = \begin{cases}2 \frac{\min \{x, y\}-x y}{N} & (x, y) \in[0,1]^{2} \\
0 & \text { otherwise, }\end{cases} \\
E_{N} & =\frac{1}{3 N}+o\left(\frac{1}{N}\right) .
\end{aligned}
$$

Similar expressions have been derived, using a different approach, in Refs. [24,25] for the grid-Poisson matching problem.

Matching problem on the unit square.-Let us now consider $\Omega=\left\{\left(x_{1}, x_{2}\right) \in \mathbb{R}^{2}: 0<x_{1}<1,0<x_{2}<1\right\} \subset \mathbb{R}^{2}$. Let us suppose also that $\rho(\mathbf{x})=1$ on $\Omega$. Using Eq. (16) we can compute $C(\mathbf{x}, \mathbf{y})$ as a function of the modified Green's function of the Laplacian on the square with Neumann boundary condition $G_{s}(\mathbf{x}, \mathbf{y})$. However, it can be seen that $N C(\mathbf{x}, \mathbf{x}) \rightarrow \infty$ for $N \rightarrow \infty$ and we need to impose a regularizing cutoff to properly evaluate this quantity. Being in this case $\delta_{N} \sim(1 / \sqrt{N})$, a regularization procedure gives us [28]

$$
E_{N}=\frac{\ln N}{2 \pi N}+\frac{\gamma}{N}+o\left(\frac{1}{N}\right)
$$

for some constant $\gamma$. Note that the leading term is exactly the same obtained for the sEBMP on the two-dimensional torus [23]. In Fig. 2 we plotted the numerical results for the AOC and we compare with the previous prediction. Moreover, we compare also our numerical results with the theoretical prediction for $c(r)$, defined as the correlation function between points on the diagonals of the square as follows

$$
\mathbf{x}_{r}:=(r, r), \quad \mathbf{y}_{r}:=(r, 1-r), \quad c(r):=N C\left(\mathbf{x}_{r}, \mathbf{y}_{r}\right)
$$

Matching problem on the flat hypertorus.-Finally, we consider the domain $\Omega \equiv[0,1]^{d} \subset \mathbb{R}^{d}$ with periodic boundary conditions; i.e., we deal with the sEBMP on the flat

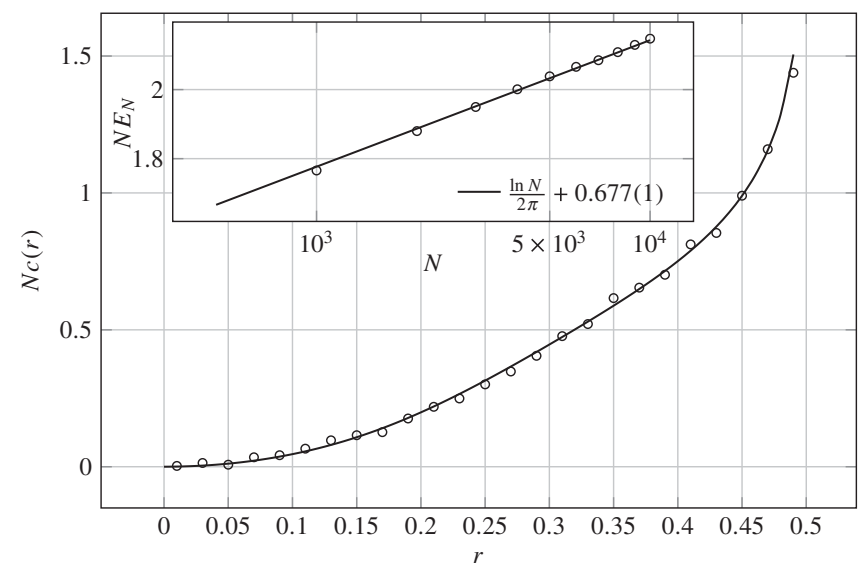

FIG. 2. Matching problem on the square. In the main plot, correlation function between points on the diagonals of the square, see Eq. (27), obtained for $N=3000$ and averaging over $2 \times 10^{4}$ instances. We compare with our analytical prediction. In the smaller plot, we compare our theoretical prediction for the AOC, Eq. (26) with numerical results obtained averaging over $2 \times 10^{4}$ instances. In particular, the value of $\gamma=0.677(1)$ is obtained by a fit procedure.

unit hypertorus in $d$ dimensions $\mathrm{T}^{d}:=\mathbb{R}^{d} / \mathbb{Z}^{d}$. We can restate the results above for this case simply by substituting the Neumann boundary conditions in Eqs. (13) and (14) with periodic boundary conditions. Moreover, the Euclidean distance in Eq. (1) between the points $\mathbf{x}=$ $\left(x_{i}\right)_{i=1, \ldots, d}$ and $\mathbf{y}=\left(y_{i}\right)_{i=1, \ldots, d}$ in $\Omega$ must be intended as

$$
\|\mathbf{x}-\mathbf{y}\|^{2}:=\sum_{i=1}^{d}\left[\min \left(\left|x_{i}-y_{i}\right|, 1-\left|x_{i}-y_{i}\right|\right)\right]^{2} .
$$

Assuming $\rho(\mathbf{x})=1$ and $\alpha=0$, then $G_{\rho}(\mathbf{x}, \mathbf{y}) \equiv G_{d}(\mathbf{x}-\mathbf{y})$, where $G_{d}(\mathbf{x}-\mathbf{y})$ is the Green's function of the Laplacian on the unit flat hypertorus $\mathrm{T}^{d}$

$$
\nabla_{\mathbf{x}}^{2} G_{d}(\mathbf{x}-\mathbf{y})=\delta^{(d)}(\mathbf{x}-\mathbf{y})-1
$$

Under these hypotheses we have that, up to higher order terms, we can formally write

$$
C(\mathbf{x}, \mathbf{y})=-\frac{2}{N} G_{d}(\mathbf{x}-\mathbf{y}), \quad E_{N}=-\frac{2}{N} G_{d}(\mathbf{0}) .
$$

For $d=1$ Eqs. (30) have the form

$$
\begin{aligned}
C(x, y) & =\frac{1-6|x-y|(1-|x-y|)}{6 N}, \\
E_{N} & =\frac{1}{6 N}+o\left(\frac{1}{N}\right)
\end{aligned}
$$

Equations (30) were adopted as a working ansatz in Refs. [23,29] and they were used to derive both the scaling of the AOC and the correlation functions of the SEBMP on 
$\mathrm{T}^{d}$. For $d \geq 2$, however, $G_{d}(\mathbf{0})$ is a divergent quantity. In this case, a nonzero value of $\alpha$ must be taken and the regularization must be performed, as shown in Ref. [23].

Conclusions.-The presented approach allows us to go beyond the mean field approximation in the SEBMP, and to easily evaluate the scaling behavior of the AOC and other useful quantities, like the correlation functions of the optimal solution. A deep connection is established among the theory of combinatorial optimization, the theory of optimal transport and the theory of disordered systems and stochastic processes. Indeed, even if optimal transport theory has been already successfully applied to many different physical problems (kinetic theory, fluidodynamics...), the study of the properties of the solution in the presence of disorder (e.g., uncertainty on the distribution parameters) is a highly nontrivial task. This interesting research line is still largely unexplored by both physicists and mathematicians and we hope that these results will allow further studies in this direction. Finally, the method presented here may be useful in the analysis of other stochastic Euclidean optimization problems, where both disorder and geometric constraints appear.

The authors are grateful to Luigi Ambrosio, Carlo Lucibello, Giorgio Parisi, and Andrea Sportiello for fruitful discussions. G. S. acknowledges the financial support of the John Templeton Foundation and the computational facilities provided by the INCT-SC.

*sergio.caracciolo@mi.infn.it †sicuro@cbpf.br; gabriele.sicuro@for.unipi.it

[1] G. Monge, Histoire de l'Académie Royale des Sciences de Paris, avec les Mémoires de Mathématique et de Physique pour la même année 666 (1781).

[2] D. Plummer and L. Lovász, Matching Theory, North-Holland Mathematics Studies (Elsevier Science, New York, 1986).

[3] C. Papadimitriou and K. Steiglitz, Combinatorial Optimization: Algorithms and Complexity, Dover Books on Computer Science (Dover, New York, 2013).

[4] H. W. Kuhn, Naval research logistics quarterly 2, 83 (1955).

[5] J. Munkres, J. Soc. Ind. Appl. Math. 5, 32 (1957).

[6] J. Edmonds and R. M. Karp, J. ACM 19, 248 (1972).
[7] R. Baxter, Exactly Solved Models in Statistical Mechanics, Dover Books on Physics Series (Dover, New York, 2007).

[8] D. Gusfield, Algorithms on Strings, Trees and Sequences: Computer Science and Computational Biology (Cambridge University Press, Cambridge, England, 1997).

[9] R. Szeliski, Computer Vision: Algorithms and Applications (Springer Science \& Business Media, New York, 2010).

[10] H. Tanaka, Z. Wahrsch. Verw. Geb. 46, 67 (1978).

[11] Y. Brenier, Commun. Pure Appl. Math. 52, 411 (1999).

[12] F. Altarelli, A. Braunstein, A. Ramezanpour, and R. Zecchina, Phys. Rev. Lett. 106, 190601 (2011).

[13] M. Mézard and A. Montanari, Information, Physics, and Computation, Oxford Graduate Texts (Oxford University Press, Oxford, 2009).

[14] M. Mézard and G. Parisi, J. Phys. (Paris), Lett. 46, 771 (1985).

[15] D. J. Aldous, Random Structures Algorithms 18, 381 (2001).

[16] S. Linusson and J. Wästlund, Probab. Theory Relat. Fields 128, 419 (2004).

[17] C. Nair, B. Prabhakar, and M. Sharma, Random Structures Algorithms 27, 413 (2005).

[18] G. Parisi, arXiv:cond-mat/9801176.

[19] D. Coppersmith and G. B. Sorkin, Random Structures Algorithms 15, 113 (1999).

[20] M. Mézard and G. Parisi, J. Phys. (Paris) 49, 2019 (1988).

[21] J. H. Boutet de Monvel and O. C. Martin, Phys. Rev. Lett. 79, 167 (1997).

[22] J. Houdayer, J. Boutet de Monvel, and O. Martin, Eur. Phys. J. B 6, 383 (1998).

[23] S. Caracciolo, C. Lucibello, G. Parisi, and G. Sicuro, Phys. Rev. E 90, 012118 (2014).

[24] E. Boniolo, S. Caracciolo, and A. Sportiello, J. Stat. Mech. (2014) P11023.

[25] S. Caracciolo and G. Sicuro, Phys. Rev. E 90, 042112 (2014).

[26] C. Villani, Optimal Transport: Old and New, Grundlehren der mathematischen Wissenschaften (Springer, New York, 2008).

[27] L. Ambrosio, Lecture Notes on Optimal Transport Problems (Springer, New York, 2003).

[28] See the Supplemental Material at http://link.aps.org/ supplemental/10.1103/PhysRevLett.115.230601 for a derivation of the correlation function and the scaling of the AOC for this case.

[29] S. Caracciolo and G. Sicuro, Phys. Rev. E 91, 062125 (2015). 